\title{
A General Approach of Quasi-Exactly Solvable Schrödinger Equations
}

\author{
N. DEBERGH円(a), J. NDIMUBANDI'(b), \\ and B. VAN DEN BOSSCHE[(a)
}

(a)Fundamental Theoretical Physics, Institute of Physics (B5), University of Liège, B-4000 LIEGE (Belgium)

(b) University of Burundi, Department of Mathematics, P.O. Box 2700, BUJUMBURA (Burundi)

\begin{abstract}
We construct a general algorithm generating the analytic eigenfunctions as well as eigenvalues of one-dimensional stationary Schrödinger Hamiltonians. Both exact and quasi-exact Hamiltonians enter our formalism but we focus on quasi-exact interactions for which no such general approach has been considered before. In particular we concentrate on a generalized sextic oscillator but also on the Lamé and the screened Coulomb potentials.
\end{abstract}

\footnotetext{
${ }^{1}$ Email: Nathalie.Debergh@ulg.ac.be

${ }^{2}$ Email: jndimubandi@yahoo.fr

${ }^{3}$ Email: bvandenbossche@ulg.ac.be
} 


\section{Introduction}

Quasi-exactly solvable (Q.E.S.) Schrödinger equations nowadays attract much attention. They are equations for which a finite number of solutions can be analytically determined. In a sense, they constitute the intermediate step between exactly solvable equations (associated with potentials such as the harmonic oscillator one, the Coulomb one,...) whose all analytic solutions can be obtained, and the analytically unsolvable ones requiring a numerical treatment. These Q.E.S. equations have been studied following two different points of view.

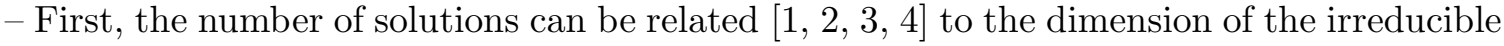
representations of a Lie algebra, namely $s l(2, R)$. In that case the corresponding Schrödinger Hamiltonian can be written as linear and quadratic combinations of the $s l(2, R)$ generators, these ones preserving the finite-dimensional space of the solutions. Due to the preservation of this space, the associated time-independent differential Schrödinger equation actually reduces to an algebraic one.

- Second, this reduction from differential to algebraic is maintained but the number of known solutions (essentially two [5, 6] or three [7]) is fixed at the start. Generally speaking this second point of view is not subtended by any Lie algebra except in the case of the two solutions [8]. Instead, it is relevant of supersymmetric quantum mechanics [9].

The main purpose of this paper is to propose a general approach unifying these two points of view. Our approach, which is also algebraic and, for the moment, restricted to the real line ] $-\infty, \infty$ [, or half line ]0, $\infty$ [, is well-suited for all potentials leading to a solution given by an overall function modulated by a polynomial. To our knowledge, it reproduces all the known exact cases which can be expressed in terms of polynomials, and most, if not all, of Q.E.S. models. For example, for the exact cases, it reproduces solutions of the harmonic oscillator, the Morse and Pöschl-Teller potentials, solutions of the Lamé equation [10], the even potentials of the class described recently by Bender and Wang in Ref. [11. On the contrary, since the odd potentials of the latter class cannot be written in term of polynomials (the solution is written as a non-terminating confluent hypergeometric function), they cannot be dealt with. As examples of Q.E.S. Schrödinger equations, we can quote all the potentials given by Turbiner in [3], a variation on the Lamé equation as described in [12], as well as the Tkachuk potential of Ref. [5]. The list is non-exhaustive.

The paper is organized as follows: In Section 2, we explain the formalism of our method in a simplifying limit regarding the expansion basis. In Section 3, we develop the most general algorithm based on a single basis function, and restricted to finite sums. In Section 4, we show the relevance of the method by investigating several well-known Q.E.S. equations, of both types quoted above. We show in Section 5 that it can be applied to yet unused potential by considering a modification of the sextic oscillator potential. Finally, we draw our conclusions in Section 6 . 


\section{A Q.E.S. general approach}

As stated in the Introduction, we concentrate on the one-dimensional and time-independent Schrödinger equation

$$
\left[-\frac{d^{2}}{d x^{2}}+V(x)\right] \psi_{N}(x)=E_{N} \psi_{N}(x), N=0,1,2, \ldots
$$

for which a finite number $(=n+1)$ of solutions $\psi_{N}(x)$ can be determined. The procedure we shall explain here is not the most general one, which can be found in Section 3. We proceed so because we want to give here more details relevant to understand the philosophy of our approach. In order to cover the Lie algebraic and non-Lie algebraic Q.E.S. equations we write the potential $V(x)$ as

$$
V(x)=\sum_{k=-2}^{V \max } V_{k}[f(x)]^{k},
$$

$V \max$ being a positive integer, while the coefficients $V_{k}$ and the function $f(x)$ are, for the moment, arbitrary. Making the sum begin with the index $k=-2$ allows to take care of the centrifugal barrier in the radial equation of a 3-dimensional problem. We shall see in Eqs. (11) and (13) that the minimal value of $k$ has anyway to be $k=-2$ in order to factor out a common power of $f(x)$.

The eigenfunctions $\psi_{N}(x)$ are assumed to be

$$
\psi_{N}(x)=g(x)[f(x)]^{\lambda_{N}} \sum_{m=0}^{N} c_{m}^{(N)}[h(x)]^{m} .
$$

The function $g(x)$ plays the role of a weight factor, $[f(x)]^{\lambda_{N}}$ is introduced due to eventual singularities in (2), and the quantities $c_{m}^{(N)}$ are expansion coefficients on the basis $h(x)$. We introduce the notation

$$
\psi_{N}(x)=\sum_{m=0}^{N} c_{m}^{(N)} \psi_{m}(x)
$$

with

$$
\psi_{m}(x)=g(x)[f(x)]^{\lambda_{N}}[h(x)]^{m}
$$

We then have

$$
\begin{aligned}
& {\left[-\frac{d^{2}}{d x^{2}}+V(x)\right] \psi_{m}(x)=\left\{-\frac{g^{\prime \prime}(x)}{g(x)}-\lambda_{N} \frac{f^{\prime \prime}(x)}{f(x)}-m \frac{h^{\prime \prime}(x)}{h(x)}\right.} \\
& -\lambda_{N}\left(\lambda_{N}-1\right) \frac{f^{\prime 2}(x)}{f^{2}(x)}-m(m-1) \frac{h^{\prime 2}(x)}{h^{2}(x)}-2 \lambda_{N} \frac{f^{\prime}(x) g^{\prime}(x)}{f(x) g(x)} \\
& \left.-2 m \frac{g^{\prime}(x) h^{\prime}(x)}{g(x) h(x)}-2 m \lambda_{N} \frac{f^{\prime}(x) h^{\prime}(x)}{f(x) h(x)}+\sum_{k=-2}^{V \max } V_{k}[f(x)]^{k}\right\} \psi_{m}(x),
\end{aligned}
$$

where the prime stands for the derivative with respect to $x$. The key idea for ensuring the quasi-exact solvability of (1) is to stabilize the space of $\psi_{m}(x)(m=0,1, \ldots, N)$, or in other 
words to ask for the second member of (6) to be a linear combination of the $\psi_{m}(x)$. Because of the different dependences of the terms of this second member (with respect to $m$, etc.), we ensure the quasi-exact solvability by requiring

$$
\begin{aligned}
f(x) & =\sum_{l=0}^{M} f_{l}^{0}[h(x)]^{l}, \\
f^{\prime}(x) & =\sum_{l=0}^{M} f_{l}^{1}[h(x)]^{l}, \\
g^{\prime}(x) & =-g(x) \sum_{l=0}^{M} g_{l}^{1}[h(x)]^{l}, \\
h^{\prime}(x) & =\sum_{l=0}^{M} h_{l}^{1}[h(x)]^{l},
\end{aligned}
$$

the different coefficients $f_{l}^{0}, f_{l}^{1}, g_{l}^{1}, h_{l}^{1}$ having to be fixed according to the potentials we have to consider. The upper-right index indicates the derivative order of the corresponding expanded function, e.g., to $f(x)$ correspond the expansion coefficients $f_{l}^{0}$, while to $f^{\prime}(x)$ correspond the coefficients $f_{l}^{1}$. The upper summation index $M$ might be different for $f(x), f^{\prime}(x), g^{\prime}(x), h^{\prime}(x)$. For simplicity, we take the same value: It is simply the highest value of the three different indices, the added expansion coefficients being vanishing. For example, if the sum leading to $f(x)$ in Eq. (17) is over the range $l \in[0, P]$ with $P<M$, we can extend it to the range $l \in[0, M]$ with $f_{i}^{0}=0$ for $i \in[P+1, M]$. Since $h(x)$ is a function to be chosen from the beginning, $f^{\prime}(x)$ and $h^{\prime}(x)$ are known. One just has to consider $M$ big enough in order to be able to determine $g(x)$.

Taking care of (8)-(10), we can rewrite (6) as

$$
\begin{aligned}
& {\left[-\frac{d^{2}}{d x^{2}}+V(x)\right] \psi_{m}(x)=\left[-\sum_{i, j} g_{j}^{1} g_{i-j}^{1}+\sum_{i, j}(i-j+1) h_{j}^{1} g_{i-j+1}^{1}\right.} \\
& \left.-m \sum_{i, j} j h_{j}^{1} h_{i-j+2}^{1}-m(m-1) \sum_{i, j} h_{j}^{1} h_{i-j+2}^{1}+2 m \sum_{i, j} h_{j}^{1} g_{i-j+1}^{1}\right] \\
& \times g(x)[f(x)]^{\lambda_{N}}[h(x)]^{m+i}+\left[-\lambda_{N} \sum_{i, j}(i-j+1) h_{j}^{1} f_{i-j+1}^{1}\right. \\
& \left.+2 \lambda_{N} \sum_{i, j} f_{j}^{1} g_{i-j}^{1}-2 m \lambda_{N} \sum_{i, j} h_{j}^{1} f_{i-j+1}^{1}\right] g(x)[f(x)]^{\lambda_{N}-1}[h(x)]^{m+i} \\
& +\left[-\lambda_{N}\left(\lambda_{N}-1\right) \sum_{i, j} f_{j}^{1} f_{i-j}^{1}\right] g(x)[f(x)]^{\lambda_{N}-2}[h(x)]^{m+i} \\
& +\sum_{k} V_{k} g(x)[f(x)]^{\lambda_{N}+k}[h(x)]^{m},
\end{aligned}
$$

where we have used

$$
\sum_{i=0}^{\infty} a_{i} \sum_{j=0}^{\infty} b_{j}=\sum_{i=0}^{\infty} \sum_{j=0}^{i} b_{j} a_{i-j}
$$


and where, for simplicity, we have omitted all the summation indices't They will anyway not be needed in the general procedure to be described in the next Section. For the moment, we have not yet considered the expansion (7). It is used in (11) when extracting a common factor $f(x)^{\lambda_{N}-2}$ :

$$
\left[-\frac{d^{2}}{d x^{2}}+V(x)\right] \psi_{m}(x)=\sum_{i} h_{m, m+i}^{(N)} g(x)[f(x)]^{\lambda_{N}-2}[h(x)]^{m+i}
$$

with

$$
\begin{aligned}
& h_{m, m+i}^{(N)} \equiv-\lambda_{N}\left(\lambda_{N}-1\right) \sum_{j} f_{j}^{1} f_{i-j}^{1}-\lambda_{N} \sum_{j, l_{1}}\left(i-j-l_{1}+1\right) h_{j}^{1} f_{i-j-l_{1}+1}^{1} f_{l_{1}}^{0} \\
& +2 \lambda_{N} \sum_{j, l_{1}} f_{j}^{1} g_{i-j-l_{1}}^{1} f_{l_{1}}^{0}-2 m \lambda_{N} \sum_{j, l_{1}} h_{j}^{1} f_{i-j-l_{1}+1}^{1} f_{l_{1}}^{0} \\
& -\sum_{j, l_{1}, l_{2}} g_{j}^{1} g_{i-j-l_{1}-l_{2}}^{1} f_{l_{1}}^{0} f_{l_{2}}^{0}+\sum_{j, l_{1}, l_{2}}\left(i-j-l_{1}-l_{2}+1\right) h_{j}^{1} g_{i-j-l_{1}-l_{2}+1}^{1} f_{l_{1}}^{0} f_{l_{2}}^{0} \\
& -m \sum_{j, l_{1}, l_{2}} j h_{j}^{1} h_{i-j-l_{1}-l_{2}+2}^{1} f_{l_{1}}^{0} f_{l_{2}}^{0}-m(m-1) \sum_{j, l_{1}, l_{2}} h_{j}^{1} h_{i-j-l_{1}-l_{2}+2}^{1} f_{l_{1}}^{0} f_{l_{2}}^{0} \\
& +2 m \sum_{j, l_{1}, l_{2}} h_{j}^{1} g_{i-j-l_{1}-l_{2}+1}^{1} f_{l_{1}}^{0} f_{l_{2}}^{0} \\
& +\sum_{k, l_{1}, l_{2}, \ldots l_{k+1}} V_{k} f_{l_{1}}^{0} f_{l_{2} \ldots f_{l_{k+1}}^{0} f_{i-l_{1}-l_{2}-\ldots-l_{k+1}}^{0} \cdot}
\end{aligned}
$$

We thus have

$$
\left[-\frac{d^{2}}{d x^{2}}+V(x)\right] \psi_{N}(x)=\sum_{m, i} c_{m}^{(N)} h_{m, m+i}^{(N)} g(x)[f(x)]^{\lambda_{N}-2}[h(x)]^{m+i}
$$

which, according to (11), must be equal to

$$
E_{N} \psi_{N}(x)=\sum_{m} c_{m}^{(N)} E_{N} \sum_{l_{1}, i} f_{l_{1}}^{0} f_{i-l_{1}}^{0} g(x)[f(x)]^{\lambda_{N}-2}[h(x)]^{m+i} .
$$

Equating the respective second members of (15) and (16), we are led to the final algebraic equations, valid for all possible values of $j$, giving rise to $\psi_{N}(x)$, as well as $E_{N}$ :

$$
\sum_{m=0}^{N} c_{m}^{(N)}\left(h_{m, j}^{(N)}-E_{N} \sum_{l_{1}, l_{2}} f_{l_{1}}^{0} f_{l_{2}}^{0} \delta_{m, j-l_{1}-l_{2}}\right)=0,
$$

where, let us recall it, $h_{m, j}$ is defined through (14). We have thus reformulated the problem (11) to the form (17). The latter looks heavy to handle. However, it can be straightforwardly implemented into a symbolic computer program.

Before turning to the general procedure, let us finally notice that the approach developed in this Section reduces to the one which can be found in [13] if $V_{-1}=V_{-2}=0, \lambda_{N}=0$ and $f(x)=h(x)$.

\footnotetext{
${ }^{4}$ The relation between the coefficients $f_{l}^{0}$ and $f_{l}^{1}$ of Eqs. (7) and (8) can be obtained with the help of Eqs. (10) and (12). This relation is $f_{l}^{1}=\sum_{k} h_{k} f_{l+1-k}^{0}(l+1-k)$.
} 


\section{A more general approach}

In this Section, we briefly describe the most general algorithm we have developped starting from the approach of the previous Section. As far as we now, there is no extension of it if we restrict ourselves to one basis function $h(x)$. A generalization to more basis functions $\left(h_{1}(x), h_{2}(x), \ldots\right)$ is possible. In particular, some of the potentials we have studied may be treated using this generalization. However, they may as well be treated using the algorithm we present below. For this reason, the study of the generalized algorithm based on more basis functions is left for a future work.

In the previous Section, we have described in details the philosophy of our approach to find analytical solutions to a given Schrödinger equation. Those analytical solutions are possible if we can factor out appropriate powers of $h(x)$ and $f(x)$, thus closing the system, see Eq. (13). This later equation was found using the expansion of the potential on a basis $f(x)$, see Eq. (2), together with the assumptions (7) $-(10)$. We show now how to relax these constraints in order to treat the maximum number of different potentials.

The simplest extension is to allow negative indices for the sums in Eqs. (7)-(10). This brings no difficulties, and this is why we did not specified the range of the indices in the previous Section:

$$
\sum_{i=-\infty}^{\infty} a_{i} \sum_{j=-\infty}^{\infty} b_{j}=\sum_{i=-\infty}^{\infty} \sum_{j=-\infty}^{\infty} b_{j} a_{i-j} \equiv \sum_{i} \sum_{j} b_{j} a_{i-j},
$$

where, from now on, an index without range means a running on $\mathbb{Z}$. Taking care of negative indices is required for example when using a basis such as $h(x)=\exp (-x)$. One can understand that to obtain a solution starting like $\cosh (x)$ may require negative powers of $h(x): 2 \cosh (x)=$ $h(x)+h(x)^{-1}$. For the same reason, the expansion of (3i) is extended to start from negative orders.

To allow more flexibility in the choice of the potential $V(x)$ in (2), it is also necessary to make the sum begining to a lower negative index than $k=-2$. For this reason, we make it begin at $k=-V \min$, with the condition $V \min \geq 2$ this restriction being due to the fact that at least a factor of $f(x)^{\lambda_{N}-2}$ must be factored out in order to close the system.

In studying the way the different powers of $h(x)$ enter into Eq. (6), we can also convince ourselves that integer powers of the square root of the right-hand-side of Eq. (10) can be chosen.

The analysis can be done using an arbitray power $\alpha$ : $h^{\prime}(x)=\left\{\sum_{l=-\infty}^{\infty} h_{l}^{1}[h(x)]^{l}\right\}^{\alpha}$. Plugging this expansion into (6), it is straighforward to see that the condition $2 \alpha=n \in \mathbb{N}$ is required. Moreover, using (18) and properly redefining the expansion coefficients, we can always write this $h^{\prime}(x)$ as the product of a series of the form of (10) with a series of the form of the square root of (10).

Using a square root in $h^{\prime}(x)$ also requires to generalize the term $g(x)$ in order, for example, to treat properly the term $g^{\prime}(x) h^{\prime}(x)$ in Eq. (6). The generalization consists in using the exponential of a $h(x)$-series, instead of the differential form given in (9), or, equivalently, to multiply the latter by $h^{\prime}(x)$ and properly redefine the expansion coefficients.

To summarize, the different expansions replacing Eqs. (2), (7) and (9)-(10) are:

$$
\psi_{N}(x)=\sum_{m=-N}^{N} c_{m}^{(N)} \psi_{m}(x)
$$




$$
\begin{aligned}
V(x) & =\sum_{k=-V \min }^{V \max } V_{k}[f(x)]^{k}, \\
f(x) & =\sum_{l} f_{l}^{0}[h(x)]^{l}, \\
g(x) & =\exp \left\{-\sum_{l} \tilde{g}_{l}^{1}[h(x)]^{l}-\tilde{g}_{\log }^{1} \ln [h(x)]\right\}, \\
h^{\prime}(x) & =\left\{\sum_{l} h_{l}^{1}[h(x)]^{l}\right\}\left\{\sum_{l} \tilde{h}_{l}^{1}[h(x)]^{l}\right\}^{1 / 2},
\end{aligned}
$$

where we have added a term $\tilde{g}_{\log }^{1} \ln [h(x)]$ in Eq. (22). This is equivalent to multiplying Eq. (3)

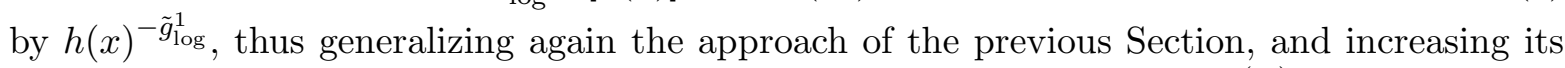
flexibility. We recover the algorithm of the previous Section by taking $c_{m<0}^{(N)}=0, \tilde{h}_{l}^{1}=\delta_{l, 0}$, $\tilde{g}_{\log }^{1}=0, g_{l}^{1}=\sum_{n} h_{n}^{1} \tilde{g}_{l-n+1}^{1}(l-n+1)$. We also remark that not all the unknowns are independent: For example, a problem with $N=1, \tilde{g}_{\mathrm{log}}^{1}=0$ implies the sum, see Eq. (19), $c_{-1}^{(1)} h(x)^{-1}+c_{0}^{(1)}+c_{1}^{(1)} h(x)$. This is equivalent to $h(x)^{-1}\left[c_{-1}^{(1)}+c_{0}^{(1)} h(x)+c_{1}^{(1)} h(x)^{2}\right]$. A similar result is achieved with $N=2, \tilde{g}_{\log }^{1}=1, c_{-2}^{(2)}=c_{-1}^{(2)}=0, c_{0}^{(2)}=c_{-1}^{(1)}, c_{1}^{(2)}=c_{0}^{(1)}, c_{2}^{(2)}=c_{1}^{(1)}$.

The last possible generalization we quote is to modify again the expansion of the potential. The function $f(x)$ in Eq. (20) can be modulated by any linear combination of the basis function $h(x)$. Indeed, with new coefficients $v_{l, k}$ of two indices refering to the power $k$ of $f(x)$ and to the power $l$ of $h(x)$, the potential

$$
V(x)=\sum_{k=-V \min }^{V \max } V_{k}[f(x)]^{k} \sum_{l} v_{l, k}[h(x)]^{l},
$$

when multiplied by $\psi_{m}(x)$ of Eq. (5) just leads to the factorization of a power of $h(x)$. This is seen as follows:

$$
\begin{aligned}
& V(x) \psi_{m}(x)= \\
& \sum_{k=0}^{V \min +V \max } V_{k-V \min } f(x)^{k} \sum_{l} v_{l, k-V \min }[h(x)]^{l} g(x)[h(x)]^{m} f(x)^{\lambda_{N}-V \min } \\
= & \sum_{k=0}^{V \min +V \max } V_{k-V \min } \sum_{n}{ }^{k} f_{n}^{0}[h(x)]^{n} \sum_{l} v_{l, k-V \min }[h(x)]^{l} g(x)[h(x)]^{m} f(x)^{\lambda_{N}-V \min } \\
= & \sum_{k=0}^{V \min +V \max } V_{k-V \min } \sum_{l}{ }^{k} \tilde{f}_{l}^{0}[h(x)]^{l+m} g(x) f(x)^{\lambda_{N}-V \min },
\end{aligned}
$$

where we have used - compare with the last line of Eq. (14) -

$$
f(x)^{k}=\left\{\sum_{l} f_{l}^{0}[h(x)]^{l}\right\}^{k}=\sum_{l}{ }^{k} f_{l}^{0}[h(x)]^{l},
$$

and where 


$$
\begin{aligned}
\sum_{n}{ }^{k} f_{n}^{0}[h(x)]^{n} \sum_{l} v_{l, k-V \min }[h(x)]^{l} & =\sum_{l}[h(x)]^{l} \sum_{n}{ }^{k} f_{n}^{0} v_{l-n, k-V \min } \\
& =\sum_{l}{ }^{k} \tilde{f}_{l}^{0}[h(x)]^{l},
\end{aligned}
$$

the last equality defining the coefficient ${ }^{k} \tilde{f}_{l}^{0}$.

In Eq. (26), the coefficients ${ }^{k} f_{l}^{0}$ are defined recursivelly:

$$
f(x)^{k}=f(x) f(x)^{k-1} \Longrightarrow{ }^{k} f_{l}^{0}=\sum_{n}{ }^{k-1} f_{l-n}^{0} f_{n}^{0}
$$

with the starting coefficient ${ }^{0} f_{l}^{0}=\delta_{l, 0}$. In defining the different coefficients, we have taken the convention that the upper-left index refers to the power of the given function, while, as in the previous Section, the upper-right index indicates the order of its derivative. (We do not, however, specify the upper-left index when it is equal to 1 , e.g., ${ }^{1} f_{l}^{i} \equiv f_{l}^{i}$.)

With the generalization given in Eqs. (21)-(24), and with the definitions of the coefficients in (27)-(28), we are now prepared to give the full algorithm solving Eq. (11). For this, we introduce also the following notation for the different terms entering Eq. (6):

$$
\begin{aligned}
{ }^{2} h_{l}^{1} & \equiv \sum_{m} h_{m}^{1} \tilde{h}_{l-m}^{1}, \\
{\left[h^{\prime}(x)\right]^{2} } & =\sum_{l}[h(x)]^{l} \sum_{m}{ }^{2} h_{m}^{1} \tilde{h}_{l-m}^{1} \equiv \sum_{l}{ }^{2} h_{l}^{1}[h(x)]^{l}, \\
f^{\prime}(x) & =\sum_{l} f_{l+1}^{0}(l+1)[h(x)]^{l} h^{\prime}(x) \equiv \sum_{l} \tilde{f}_{l}^{1}[h(x)]^{l} h^{\prime}(x), \\
{ }^{2} \tilde{f}_{l}^{1} & \equiv \sum_{m} \tilde{f}_{m}^{1} \tilde{f}_{l-m}^{1}, \\
{\left[f^{\prime}(x)\right]^{2} } & =\sum_{l}[h(x)]^{l} \sum_{m}{ }^{2} h_{m}^{1}{ }^{2} \tilde{f}_{l-m}^{1} \equiv \sum_{l}{ }^{2} f_{l}^{1}[h(x)]^{l}, \\
g^{\prime}(x) & =-g(x)\left\{\sum_{l} \tilde{g}_{l+1}^{1}(l+1)[h(x)]^{l}+\tilde{g}_{\text {log }}^{1} / h(x)\right\} h^{\prime}(x) \\
& \equiv-g(x) h^{\prime}(x) \sum_{l} g_{l}^{1}[h(x)]^{l}, \\
{ }^{2} \tilde{g}_{l}^{1} & \equiv \sum_{m} g_{m}^{1} g_{l-m}^{1}, \\
{\left[g^{\prime}(x)\right]^{2} } & =[g(x)]^{2} \sum_{l}[h(x)]^{l} \sum_{m}{ }^{2} h_{m}^{1} \tilde{g}_{l-m}^{1} \equiv g(x)^{2} \sum_{l}{ }^{2} g_{l}^{1}[h(x)]^{l}, \\
f^{\prime}(x) h^{\prime}(x) & =\sum_{l}[h(x)]^{l} \sum_{m}{ }^{2} h_{m}^{1} \tilde{f}_{l-m}^{1} \equiv \sum_{l} f h_{l}^{1}[h(x)]^{l}, \\
g^{\prime}(x) h^{\prime}(x) & =-g(x) \sum_{l}[h(x)]^{l} \sum_{m}{ }^{2} h_{m}^{1} g_{l-m}^{1} \equiv \sum_{l} g h_{l}^{1}[h(x)]^{l}, \\
f^{\prime}(x) g^{\prime}(x) & =-g(x)\left[h^{\prime}(x)\right]^{2} \sum_{l}[h(x)]^{l} \sum_{m} g_{m}^{1} \tilde{f}_{l-m}^{1} \equiv-g(x)\left[h^{\prime}(x)\right]^{2} \sum_{l} \tilde{f}_{l}^{1}[h(x)]^{l}
\end{aligned}
$$




$$
\begin{aligned}
& =-g(x) \sum_{l}[h(x)]^{l} \sum_{m} \tilde{f}_{m}^{1}{ }^{2} h_{l-m}^{1} \equiv-g(x) \sum_{l} f g_{l}^{1}[h(x)]^{l}, \\
\tilde{\tilde{h}}_{l}^{1} & \equiv \sum_{m} h_{m+1}^{1} h_{l-m}^{1}, \\
h^{\prime \prime}(x) & =\sum_{l}[h(x)]^{l} \sum_{m}\left[\tilde{\tilde{h}}_{m}^{1} \tilde{h}_{l-m}^{1}+\frac{1}{2} \tilde{h}_{m+1}(m+1)^{2} h_{l-m}^{1}\right] \\
& \equiv \sum_{l} h_{l}^{2}[h(x)]^{l}, \\
f^{\prime \prime}(x) & =\sum_{l}[h(x)]^{l} \sum_{m}\left[{ }^{2} h_{m}^{1} \tilde{f}_{l+1-m}^{1}(l+1-m)+h_{m}^{2} \tilde{f}_{l-m}^{1}\right] \\
& \equiv \sum_{l} f_{l}^{2}[h(x)]^{l}, \\
g^{\prime \prime}(x) & =g(x) \sum_{l}[h(x)]^{l}\left[2 g_{l}^{1}-\sum_{m} g_{m+1}^{1}(m+1)^{2} h_{l-m}^{1}-\sum_{m} g_{m}^{1} h_{l-m}^{2}\right] \\
& \equiv g(x) \sum_{l} g_{l}^{2}[h(x)]^{l},
\end{aligned}
$$

where the equivalence signs in the above equations define new coefficients. In obtaining these, we have made repetive use of (18). Note that the coefficient $g_{l}^{1}$ of Eq. (34) is defined differently from (9). These notations are quite lengthy. However, they allow to obtain an algebraic equation very suitable for a symbolic computer program, generalization of Eq. (17) with (14):

$$
\begin{aligned}
\sum_{m=-N}^{N} c_{m}^{(N)}[ & \left.h_{m, j}^{(N)}-E_{N}\left({ }^{V \min } f_{j-m}^{0}\right)\right]=0 \\
h_{m, j}^{(N)} \equiv-\sum_{n} & {\left[\lambda_{N}\left(\lambda_{N}-1\right)\left({ }^{V \min -2} f_{n}^{0}\right)\left({ }^{2} f_{j-m-n}^{1}\right)+\lambda_{N}\left({ }^{V \min -1} f_{n}^{0}\right) f_{j-m-n}^{2}\right.} \\
& -2 \lambda_{N}\left({ }^{V \min -1} f_{n}^{0}\right) f g_{j-m-n}^{1}+2 m \lambda_{N}\left({ }^{V \min -1} f_{n}^{0}\right) f h_{j-m-n+1}^{1} \\
& +\left({ }^{V \min } f_{n}^{0}\right) g_{j-m-n}^{2}+m\left({ }^{V \min } f_{n}^{0}\right) h_{j-m-n+1}^{2} \\
& \left.+m(m-1)\left({ }^{V \min } f_{n}^{0}\right)\left({ }^{2} h_{j-m-n+2}^{1}\right)-2 m\left({ }^{V \min } f_{n}^{0}\right) g h_{j-m-n+1}^{1}\right] \\
& +\sum_{k=0}^{V \min +V \max } V_{k-V \min }\left({ }^{k} \tilde{f}_{j-m}^{0}\right) .
\end{aligned}
$$

This algebraic equation has to be solved for each value of $j$. Apart from the possible generalization using several basis functions $\left(h_{1}(x), h_{2}(x), \cdots\right)$ that we mentioned in the beginning of this Section, this equation is the most general algorithm we have found to solve Eq. (11).

In the next Section, we present several examples using both the generalized algorithm (45)(44) and the simplest one given by Eqs. (14) and (17). We use the latter when that the

\footnotetext{
${ }^{5}$ A slight generalization is possible, consisting in taking, instead of the parametrization (24) of the potential, the ratio of two such paramatrizations. We do not consider this generalization here because we did not find an example needing it. This does not, however, exclude this possibility.
} 
generalizations are not needed because it works faster. The examples are treated using a Mathematica implementation of Eqs. (14), (17), (45) and (44).

\section{Examples}

In this Section, we prove the convenience of our method for the determination of the eigenvalues and eigenfunctions of well-known Q.E.S. equations. We start with the genelarized sextic oscillator. We begin with a general set of parameters and show by fixing some of these parameters that the constraints between the different left parameters can be solved. In order to illustrate our method, a lot of details are given in the first treatment of the problem. Then, as we go on, only the main points are stressed.

\subsection{The generalized sextic oscillator}

The generalized sextic potential has the form

$$
\begin{aligned}
V(x)= & V_{3} b^{3} x^{6}+\left(3 V_{3} a b^{2}+V_{2} b^{2}\right) x^{4}+\left(3 V_{3} a^{2} b+2 V_{2} a b+V_{1} b\right) x^{2} \\
& +V_{3} a^{3}+V_{2} a^{2}+V_{1} a+V_{0}+\frac{V_{-1}}{a+b x^{2}}+\frac{V_{-2}}{\left(a+b x^{2}\right)^{2}}
\end{aligned}
$$

with $b V_{3} \geq 0$ and $a b \geq 0$. It coincides with the sextic radial oscillator analyzed by Ushveridze [4] if

$$
\begin{aligned}
& V_{3}=t^{2}, V_{2}=0, V_{1}=-4 t\left(s+\frac{1}{2}+n\right), V_{0}=0, \\
& V_{-1}=4\left(s-\frac{1}{4}\right)\left(s-\frac{3}{4}\right), V_{-2}=0, a=0, b=1,
\end{aligned}
$$

while the Tkachuk potential [5] is recovered for

$$
V_{3}=\frac{1}{4 b}, V_{2}=-\frac{a}{4 b}, V_{1}=-3, V_{0}=\frac{5}{2} a, V_{-1}=\frac{3}{4} b, V_{-2}=\frac{3}{4} a b
$$

in the context of two solutions.

We see from (46) that $V(x)$ can be written as Eq. (2) with the identification

$$
M=3, f(x)=a+b x^{2} .
$$

This, as well as $f^{\prime}(x)=2 b x$, clearly suggests the basis function $h(x) \equiv x$. From the relations (7)-(10) we get the nonvanishing coefficients:

$$
f_{0}^{0}=a, f_{2}^{0}=b, f_{1}^{1}=2 b, h_{0}^{1}=1 .
$$

The unknowns of the problem are $E_{N}, \lambda_{N}, c_{m}^{(N)}, g_{l}^{1}$. They have to be found from Eq. (17). Studying the case $N=0$, we can convince ourselves that the only nonvanishing coefficients of the weight function $g(x)$ are $g_{l}^{1}(l=0,1,2,3)$. Indeed, Eq. (17) reduces to

$$
h_{0, j}^{(0)}-E_{0}\left(a^{2} \delta_{j, 0}+2 a b \delta_{j, 2}+b^{2} \delta_{j, 4}\right)=0, j=0,1, \ldots, 10
$$


with-see Eq. (14) -

$$
\begin{aligned}
h_{0, j}^{(0)}= & {\left[-2 a b \lambda_{0}-a^{2}\left(g_{0}^{1}\right)^{2}+a^{2} g_{1}^{1}+V_{-2}+a V_{-1}+a^{2} V_{0}+a^{3} V_{1}+a^{4} V_{2}+a^{5} V_{3}\right] \delta_{j, 0} } \\
& +\left(4 a b \lambda_{0} g_{0}^{1}-2 a^{2} g_{0}^{1} g_{1}^{1}+2 a^{2} g_{2}^{1}\right) \delta_{j, 1}+\left[-4 b^{2} \lambda_{0}^{2}+2 b^{2} \lambda_{0}\right. \\
& +4 a b \lambda_{0} g_{1}^{1}-2 a^{2} g_{0}^{1} g_{2}^{1}-a^{2}\left(g_{1}^{1}\right)^{2}-2 a b\left(g_{0}^{1}\right)^{2}+3 a^{2} g_{3}^{1}+2 a b g_{1}^{1}+V_{-1} b+2 a b V_{0} \\
& \left.+3 a^{2} b V_{1}+4 a^{3} b V_{2}+5 a^{4} b V_{3}\right] \delta_{j, 2}+\left(4 a b \lambda_{0} g_{2}^{1}+4 b^{2} \lambda_{0} g_{0}^{1}\right. \\
& \left.-2 a^{2} g_{0}^{1} g_{3}^{1}-2 a^{2} g_{1}^{1} g_{2}^{1}-4 a b g_{0}^{1} g_{1}^{1}+4 a b g_{2}^{1}\right) \delta_{j, 3}+\left[4 a b \lambda_{0} g_{3}^{1}+4 b^{2} \lambda_{0} g_{1}^{1}\right. \\
& -2 a^{2} g_{1}^{1} g_{3}^{1}-a^{2}\left(g_{2}^{1}\right)^{2}-4 a b g_{0}^{1} g_{2}^{1}-2 a b\left(g_{1}^{1}\right)^{2}-b^{2}\left(g_{0}^{1}\right)^{2}+6 a b g_{3}^{1}+b^{2} g_{1}^{1}+b^{2} V_{0} \\
& \left.+3 a b^{2} V_{1}+6 a^{2} b^{2} V_{2}+10 a^{3} b^{2} V_{3}\right] \delta_{j, 4}+\left(4 b^{2} \lambda_{0} g_{2}^{1}-2 a^{2} g_{2}^{1} g_{3}^{1}\right. \\
& \left.-4 a b g_{0}^{1} g_{3}^{1}-4 a b g_{1}^{1} g_{2}^{1}-2 b^{2} g_{0}^{1} g_{1}^{1}+2 b^{2} g_{2}^{1}\right) \delta_{j, 5}+\left[4 b^{2} \lambda_{0} g_{3}^{1}-a^{2}\left(g_{3}^{1}\right)^{2}\right. \\
& -4 a b g_{1}^{1} g_{3}^{1}-2 a b\left(g_{2}^{1}\right)^{2}-2 b^{2} g_{0}^{1} g_{2}^{1}-b^{2}\left(g_{1}^{1}\right)^{2}+3 b^{2} g_{3}^{1}+b^{3} V_{1}+4 a b^{3} V_{2} \\
& \left.+10 a^{2} b^{3} V_{3}\right] \delta_{j, 6}+\left(-4 a b g_{2}^{1} g_{3}^{1}-2 b^{2} g_{0}^{1} g_{3}^{1}-2 b^{2} g_{1}^{1} g_{2}^{1}\right) \delta_{j, 7} \\
& +\left[-2 a b\left(g_{3}^{1}\right)^{2}-2 b^{2} g_{1}^{1} g_{3}^{1}-b^{2}\left(g_{2}^{1}\right)^{2}+b^{4} V_{2}+5 a b^{4} V_{3}\right] \delta_{j, 8} \\
& +\left(-2 b^{2} g_{2}^{1} g_{3}^{1}\right) \delta_{j, 9}+\left[-b^{2}\left(g_{3}^{1}\right)^{2}+b^{5} V_{3}\right] \delta_{j, 10} .
\end{aligned}
$$

With vanishing $g_{l}^{1}, l=5, \ldots$ expansion coefficients, we would have get an extra term $g_{4}^{1} \delta_{j, 11}$, implying $g_{4}^{1}=0$. Repeating this argument recursively, we see that only $g_{l}^{1}, l=0,1,2,3$ survive.

From the values $j=10,9,8,7$ we compute the weight function:

$$
\begin{aligned}
g(x) & \equiv \exp \left(-\sum_{l=1}^{4} \frac{g_{l-1}^{1}}{l} x^{l}\right) \\
& =\exp \left(-\frac{3}{4} a \sqrt{b V_{3}} x^{2}-\frac{1}{4} \sqrt{\frac{b}{V_{3}}} V_{2} x^{2}-\frac{1}{4} \sqrt{b^{3} V_{3}} x^{4}\right)
\end{aligned}
$$

while for $j=10$ we have taken the positive root $g_{3}^{1}=\sqrt{b^{3} V_{3}}$. We could have chosen the negative root. This would have lead to a nonphysical solution because it is not bounded when $x \rightarrow \infty$. We note however that unbounded solutions have their usefulness: they can be used to generate new potentials and the corresponding (bounded) eigenfunctions through Darboux transformations [14].

The index $j=6$ fixes $\lambda_{0}$. Together, the wavefunction $\psi_{0}(x)$ is determined to be

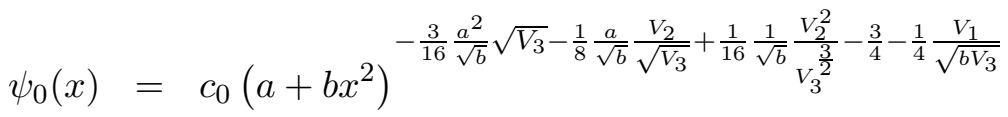

$$
\begin{aligned}
& \times \exp \left(-\frac{3}{4} a \sqrt{b V_{3}} x^{2}-\frac{1}{4} \sqrt{\frac{b}{V_{3}}} V_{2} x^{2}-\frac{1}{4} \sqrt{b^{3} V_{3}} x^{4}\right),
\end{aligned}
$$

the quantity $c_{0}$ being fixed by normalization. The next significant value $j=4$ leads to the knowledge of $E_{0}$

$$
E_{0}=\frac{5}{8} a^{3} V_{3}+\frac{3}{8} a^{2} V_{2}+\frac{1}{2} a V_{1}+V_{0}-\frac{1}{8} a \frac{V_{2}^{2}}{V_{3}}+\frac{1}{8} \frac{V_{2}^{3}}{V_{3}^{2}}-\frac{1}{2} \frac{V_{1} V_{2}}{V_{3}}-\sqrt{\frac{b}{V_{3}}} V_{2} .
$$


Finally, taking care of $j=2$ and $j=0$, we are left with two constraints on the potential (46):

$$
\begin{aligned}
& V_{-1}=\frac{1}{64} \frac{V_{2}^{4}}{V_{3}^{3}}-\frac{1}{8} \frac{V_{1} V_{2}^{2}}{V_{3}^{2}}+\frac{1}{4} \frac{V_{1}^{2}}{V_{3}}-\frac{1}{2} \sqrt{b} \frac{V_{2}^{2}}{V_{3}^{\frac{3}{2}}}+2 \sqrt{b} \frac{V_{1}}{\sqrt{V_{3}}}+\frac{15}{4} b-\frac{1}{2} a \sqrt{b} \frac{V_{2}}{\sqrt{V_{3}}} \\
& +\frac{1}{16} a \frac{V_{2}^{3}}{V_{3}^{2}}-\frac{1}{4} a \frac{V_{1} V_{2}}{V_{3}}-\frac{1}{8} a^{2} V_{1}-\frac{7}{16} a^{3} V_{2}-\frac{5}{32} a^{2} \frac{V_{2}^{2}}{V_{3}}-\frac{15}{64} a^{4} V_{3}, \\
& V_{-2}=\frac{1}{8} a \frac{V_{1} V_{2}^{2}}{V_{3}^{2}}-\frac{1}{4} a \frac{V_{1}^{2}}{V_{3}}-\frac{1}{64} a \frac{V_{2}^{4}}{V_{3}^{3}}-\frac{1}{4} a^{2} \frac{V_{1} V_{2}}{V_{3}}+\frac{1}{16} a^{2} \frac{V_{2}^{3}}{V_{3}^{2}}-\frac{9}{64} a^{5} V_{3} \\
& -\frac{3}{16} a^{4} V_{2}-\frac{3}{8} a^{3} V_{1}-\frac{5}{2} a \sqrt{b} \frac{V_{1}}{\sqrt{V_{3}}}-\frac{21}{4} a b+\frac{1}{32} a^{3} \frac{V_{2}^{2}}{V_{3}}+\frac{5}{8} a \sqrt{b} \frac{V_{2}^{2}}{V_{3}^{\frac{3}{2}}} \\
& -\frac{5}{4} a^{2} \sqrt{b} \frac{V_{2}}{\sqrt{V_{3}}}-\frac{15}{8} a^{3} \sqrt{b} \sqrt{V_{3}},
\end{aligned}
$$

respectively. The different choices (47) (with $n=0$ ) and (48) are compatible with these contraints, which are limitations on the potential: There is at least one solution to the Schrödinger equation (1) with (46), this solution being given by Eq. (54), only if the different $V_{i}, i=-2, \ldots, 3$ coefficients of the potential satisfy the constraints.

We proceed in a similar way for the $N=1$-case. Now, the set of equations (17) reads, for $j=0, \ldots, 11$ :

$$
\begin{aligned}
& c_{0}^{(1)}\left[h_{0, j}^{(1)}-E_{1}\left(a^{2} \delta_{j, 0}+2 a b \delta_{j, 2}+b^{2} \delta_{j, 4}\right)\right] \\
& \quad+c_{1}^{(1)}\left[h_{1, j}^{(1)}-E_{1}\left(a^{2} \delta_{j, 1}+2 a b \delta_{j, 3}+b^{2} \delta_{j, 5}\right)\right]=0 .
\end{aligned}
$$

The quantity $h_{0, j}^{(1)}$ is again given by Eq. (52) up to the replacement of $\lambda_{0}$ by $\lambda_{1}$, while $h_{1, j}^{(1)}$ is

$$
\begin{aligned}
& h_{1, j}^{(1)}=2 a^{2} g_{0} \delta_{j, 0}+\left[-6 a b \lambda_{1}-a^{2}\left(g_{0}^{1}\right)^{2}+3 a^{2} g_{1}^{1}+V_{-2}+a V_{-1}+a^{2} V_{0}+a^{3} V_{1}\right. \\
& \left.+a^{4} V_{2}+a^{5} V_{3}\right] \delta_{j, 1}+\left(4 a b \lambda_{1} g_{0}^{1}-2 a^{2} g_{0}^{1} g_{1}^{1}+4 a^{2} g_{2}^{1}+4 a b g_{0}^{1}\right) \delta_{j, 2} \\
& +\left[-4 b^{2} \lambda_{1}^{2}-2 b^{2} \lambda_{1}+4 a b \lambda_{1} g_{1}^{1}-2 a^{2} g_{0}^{1} g_{2}^{1}-a^{2}\left(g_{1}^{1}\right)^{2}-2 a b\left(g_{0}^{1}\right)^{2}+5 a^{2} g_{3}^{1}+6 a b g_{1}^{1}\right. \\
& \left.+V_{-1} b+2 a b V_{0}+3 a^{2} b V_{1}+4 a^{3} b V_{2}+5 a^{4} b V_{3}\right] \delta_{j, 3}+\left(4 a b \lambda_{1} g_{2}^{1}+4 b^{2} \lambda_{1} g_{0}^{1}\right. \\
& \left.\quad-2 a^{2} g_{0}^{1} g_{3}^{1}-2 a^{2} g_{1}^{1} g_{2}^{1}-4 a b g_{0}^{1} g_{1}^{1}+8 a b g_{2}^{1}+2 b^{2} g_{0}^{1}\right) \delta_{j, 4}+\left[4 a b \lambda_{1} g_{3}^{1}+4 b^{2} \lambda_{1} g_{1}^{1}\right. \\
& -2 a^{2} g_{1}^{1} g_{3}^{1}-a^{2}\left(g_{2}^{1}\right)^{2}-4 a b g_{0}^{1} g_{2}^{1}-2 a b\left(g_{1}^{1}\right)^{2}-b^{2}\left(g_{0}^{1}\right)^{2}+10 a b g_{3}^{1}+3 b^{2} g_{1}^{1}+b^{2} V_{0} \\
& \left.+3 a b^{2} V_{1}+6 a^{2} b^{2} V_{2}+10 a^{3} b^{2} V_{3}\right] \delta_{j, 5}+\left(4 b^{2} \lambda_{1} g_{2}^{1}-2 a^{2} g_{2}^{1} g_{3}^{1}\right. \\
& \left.-4 a b g_{0}^{1} g_{3}^{1}-4 a b g_{1}^{1} g_{2}^{1}-2 b^{2} g_{0}^{1} g_{1}^{1}+4 b^{2} g_{2}^{1}\right) \delta_{j, 6}+\left[4 b^{2} \lambda_{1} g_{3}^{1}-a^{2}\left(g_{3}^{1}\right)^{2}\right. \\
& -4 a b g_{1}^{1} g_{3}^{1}-2 a b\left(g_{2}^{1}\right)^{2}-2 b^{2} g_{0}^{1} g_{2}^{1}-b^{2}\left(g_{1}^{1}\right)^{2}+5 b^{2} g_{3}^{1}+b^{3} V_{1}+4 a b^{3} V_{2} \\
& \left.+10 a^{2} b^{3} V_{3}\right] \delta_{j, 7}+\left(-4 a b g_{2}^{1} g_{3}^{1}-2 b^{2} g_{0}^{1} g_{3}^{1}-2 b^{2} g_{1}^{1} g_{2}^{1}\right) \delta_{j, 8} \\
& +\left[-2 a b\left(g_{3}^{1}\right)^{2}-2 b^{2} g_{1}^{1} g_{3}^{1}-b^{2}\left(g_{2}^{1}\right)^{2}+b^{4} V_{2}+5 a b^{4} V_{3}\right] \delta_{j, 9} \\
& +\left(-2 b^{2} g_{2}^{1} g_{3}^{1}\right) \delta_{j, 10}+\left[-b^{2}\left(g_{3}^{1}\right)^{2}+b^{5} V_{3}\right] \delta_{j, 11} .
\end{aligned}
$$

The analysis of the different values of $j$ leads to the following eigenfunction

$$
\psi_{1}(x)=c_{1} \frac{x}{\left(a+b x^{2}\right)^{\frac{1}{2}}} \psi_{0}(x)
$$


corresponding to the energy

$$
E_{1}=E_{0}+2 a \sqrt{b V_{3}}
$$

iff the constraints on $V(x)$

$$
\begin{aligned}
V_{-1}(N=1)= & V_{-1}(N=0)-a \sqrt{b} \frac{V_{2}}{\sqrt{V_{3}}}-a^{2} \sqrt{b V_{3}}, \\
V_{-2}(N=1)=V_{-2}(N=0)-6 a b & -a \sqrt{b} \frac{V_{1}}{\sqrt{V_{3}}}+\frac{1}{4} a \sqrt{b} \frac{V_{2}^{2}}{V_{3}^{\frac{3}{2}}} \\
& -\frac{1}{2} a^{2} \sqrt{b} \frac{V_{2}}{\sqrt{V_{3}}}-\frac{3}{4} a^{3} \sqrt{b V_{3}}
\end{aligned}
$$

are satisfied.

We have also computed the cases $N=2,3,4$. The results are too cumbersome to be written here. We have however observed that the constraints always change with $N$ and that to $\psi_{N}(x)$ corresponds the $N$ th excited state (the identification is done looking at the number of nodes of the wavefunction). Since the constraints change with $N$, this means that each obtained level corresponds to a different potential. This is at variance with the particular case $a=0$ (see Ushveridze [4]). In this limit, we see from the comparison between Eqs. (54)-(57) and Eqs. (60)-(63) that the cases $N=0$ and $N=1$ coincide.

We also see quite straightforwardly that the constraints (56)-(57) are compatible with the parametrization given in Eq. (47), provided $n=0$. In fact, going to higher integer $n$, we can convince ourselves that there are $n+1$ solutions compatible with the constraint on $V_{-1}$. These solutions are defined on the half line $] 0, \infty\left[\right.$, due to the centrifugal barrier $V_{-1} / x^{2}$, hence the name sextic radial oscillator. The higher $n$ solutions are investigated using higher $N$ values in our algorithm.

In general, playing with the different parameters, we can generate a lot of different solutions:

- In the general case above, we mentioned the fact that to one potential corresponds a single solution;

- To the sextic radial oscillator corresponds a given number of solutions, depending on the value of the integer $n$ in (47);

- Eliminating also the centrifugal barrier of the sextic radial oscillator, $V_{-1}=0$, we are left with a model defined on ] $-\infty, \infty[$, hence the name sextic oscillator. In that case, the exact solutions fall into parity classes. To see this statement, the parametrization (47) is replaced by

$$
\begin{aligned}
& V_{3}=t^{2}, V_{2}=0, V_{1}=-4 t\left(n+\frac{p}{2}+\frac{3}{4}\right), V_{0}=0, \\
& V_{-1}=0, V_{-2}=0, a=0, b=1
\end{aligned}
$$

where $p$ denotes the parity. For a given $n$, one may get series of solutions: One with $p=0$ (even solutions), and one with $p=1$ (odd solutions). Since $p$ can take two values, the potential is then different for odd and even solutions: For a given potential, the solutions fall into parity classes, as said before; 
- Even with $V_{-1}$ and $V_{-2}$ nonvanishing, we can have an exactly solvable potential. For example, the potential

$$
V(x)=\frac{x^{2}}{4}+\frac{4}{1+x^{2}}-\frac{8}{\left(1+x^{2}\right)^{2}}
$$

is exactly solvable. This can be shown easily with our approach because we get no constraint. Theoretically, this is even more easily seen by noting that this potential can be constructed from the harmonic oscillator using a Darboux transformation [14];

- The Kuliy-Tkachuk potential [7] obtained from the general case with the special parametrization (48) is also interesting in the sense that it always generates a given number of solutions. This number is fixed from the start (for the Kuliy-Tkachuk potential, this number is three).

In the following, we shall review the different possibilities we have just enumerated.

\subsubsection{The general case}

We have already discussed the general case given by Eq. (46) for $N=0$ and $N=1$. For higher $N$, we get, before using the parametrization (47) which still has to be shown to be consistent, the condition $\lambda_{N}=\lambda_{0}-N / 2$. The equations for the constraints relating $V_{-1}$ and $V_{-2}$ to the other coefficients, and the equations for the coefficients $c_{m}^{(N)}$, relating them to a single one to be fixed by normalization, for instance $c_{0}^{(N)}$, are too complicated to be solved analytically for arbitrary $N$. However, we can find at least some of the solutions and see what they imply. Using our algorithm, one can see by direct substitution in the corresponding constraint equation that $V_{-1}^{(2 N)}=V_{-1}^{(0)} ; V_{-2}^{(2 N)}=V_{-2}^{(0)}$ and $V_{-1}^{(2 N+1)}=V_{-1}^{(1)} ; V_{-2}^{(2 N+1)}=V_{-2}^{(1)}$ are compatible constraints, for which we also observe that $E_{2 N}=E_{0}$ and $E_{2 N+1}=E_{1}$. This however does not lead to new results: In fact, we just recover the case $N=0$ and $N=1$, respectively. This is seen as follows, taking the case $N=2$ as an example: Using the constraints (56) and (57), we can solve the equation for $c_{2}^{(2)}$ as a function of $c_{0}^{(2)}$. We get $c_{2}^{(2)}=c_{0}^{(2)} b / a$. Together with the relations $\lambda_{N}=\lambda_{0}-1$ and $f(x) \equiv\left(a+b x^{2}\right)$, we have $f(x)^{\lambda_{2}}\left(c_{0}^{(2)}+c_{0}^{(2)} x^{2}\right)=f(x)^{\lambda_{0}} c_{0}^{(2)} / a$. Then, the wavefunction $\psi_{2}$ and $\psi_{0}$ are equal, up to an unimportant constant. This analysis remains true to higher $N$. This means that, when taking higher $N$, we cannot take the same constraint equations for $V_{-1}$ and $V_{-2}$ as the equations for $N=0$ or $N=1$ to get new results. This implies that the constraints on $V_{-1}$ and $V_{-2}$ are different for each value of $N$, a result that we mentioned earlier.

\subsubsection{The sextic radial oscillator}

As we indicated above, this case corresponds to the parameter $a=0$. From physical reason, we have to consider also $V_{-2}=0$. This is in fact included in the constraint equations of the general case above, see Eqs. (57) and (63). To simplify the discussion, we choose, following Ushveridze, $b=1, V_{2}=0$. As we have seen previously, the case $N=0$ and $N=1$ are degenerate. We obtain

$$
\lambda_{0} \equiv \lambda_{1}=-\frac{V_{1}+3 \sqrt{V_{3}}}{4 \sqrt{V_{3}}}
$$




$$
\begin{aligned}
E_{0} & \equiv E_{1}=0 \\
\psi_{0}(x) & \equiv \psi_{1}(x)=c_{0} \exp \left(-\frac{\sqrt{V_{3}}}{4} x^{4}\right)\left(x^{2}\right)^{-\frac{3}{4}-\frac{V_{1}}{4 \sqrt{V_{3}}}}, \\
V_{-1} & =\frac{\left(V_{1}+3 \sqrt{V_{3}}\right)\left(V_{1}+5 \sqrt{V_{3}}\right)}{4 V_{3}} .
\end{aligned}
$$

The last equation is taken from (56) or (62) with the given $a, b, V_{2}$ of this Section. It is clear that the parametrization (47) with $n=0$ satisfies it.

Let us now look the case $N=2$. We then have

$$
\begin{aligned}
\lambda_{2} & =\lambda_{0}-1 \\
E_{2} & \equiv-4 \sqrt{V_{3}} \frac{c_{0}^{(2)}}{c_{2}^{(2)}}=-\frac{4 \epsilon}{\left(V_{3}\right)^{1 / 4}} \sqrt{-\frac{1}{2} V_{1} \sqrt{V_{3}}-3 V_{3}} \\
\psi_{2}(x) & =c_{2} \exp \left(-\frac{\sqrt{V_{3}}}{4} x^{4}\right)\left(x^{2}\right)^{-\frac{7}{4}-\frac{V_{1}}{4 \sqrt{V_{3}}}}\left[1+\frac{\epsilon x^{2}\left(V_{3}\right)^{3 / 4}}{\sqrt{-\frac{1}{2} V_{1} \sqrt{V_{3}}-3 V_{3}}}\right], \\
V_{-1} & =\frac{\left(V_{1}+7 \sqrt{V_{3}}\right)\left(V_{1}+9 \sqrt{V_{3}}\right)}{4 V_{3}}
\end{aligned}
$$

where $\epsilon= \pm 1$. We see that the last equation is satisfied by (47) with $n=1$. For this constraint, two solutions are possible: A ground state with $\epsilon=1$ and a first excited state with $\epsilon=-1$. Note that the argument under the square root is non-negative. This is seen easily with the parametrization (47) with $n=1$. This can also be seen from the requirement that the wavefunctions must vanish at the origin, which implies $-7 / 4-V_{1} /\left(4 \sqrt{V_{3}}\right)>0$. This is enough to ensure a positive argument of the square root.

The case $N=3$ leads to the same results as $N=2$. For $N=4$, we get the new results

$$
\begin{aligned}
\lambda_{4} & =\lambda_{0}-2, \\
E_{4} & =-4 \sqrt{V_{3}} \frac{c_{2}^{(4)}}{c_{4}^{(4)}} \\
\psi_{4}(x) & =c_{0} \exp \left(-\frac{\sqrt{V_{3}}}{4} x^{4}\right)\left(x^{2}\right)^{-\frac{11}{4}-\frac{V_{1}}{4 \sqrt{V_{3}}}}\left[c_{0}^{(4)}+c_{2}^{(4)} x^{2}+c_{4}^{(4)} x^{4}\right], \\
V_{-1} & =\frac{\left(V_{1}+11 \sqrt{V_{3}}\right)\left(V_{1}+13 \sqrt{V_{3}}\right)}{4 V_{3}},
\end{aligned}
$$

with the three possibilities

$$
\begin{aligned}
& c_{2}^{(4)}=0, c_{4}^{(4)}=c_{0}^{(4)} \frac{2 V_{3}}{V_{1}+8 \sqrt{V_{3}}}, \\
& c_{2}^{(4)}=c_{0}^{(4)} 2 \sqrt{2} \epsilon \frac{\sqrt{\left(-V_{1}-9 \sqrt{V_{3}}\right) V_{3}}}{\left|V_{1}+10 \sqrt{V_{3}}\right|}, c_{4}^{(4)}=-2 c_{0}^{(4)} \frac{2 V_{3}}{V_{1}+10 \sqrt{V_{3}}},
\end{aligned}
$$

with $\epsilon= \pm 1$. This implies that we have three solutions corresponding to the constraint (77), which is satisfied with the parametrization (47) with $n=2$. These three solutions are the 
ground state, obtained with (79) and $\epsilon=1$, the first excited state, obtained with (78), and the second excited state, obtained with (79) and $\epsilon=-1$. The arguments under the square root are non-negative. The argument follows the same lines as in the case $n=1$.

The case $N=5$ is again identical to $N=4$.

Using our algorithm, it is easy to see, and we have already mentioned it, that $\lambda_{N}=\lambda_{0}-N / 2$, or, because odd values of $N$ give identical results to the even values, $\lambda_{2 n}=\lambda_{0}-n$ (see Eqs. (70) and (74)). This is true for arbitrary $V_{1}$ and $V_{3}$. From Eq. (66), a vanishing wavefunction at the origin implies $-V_{1} / \sqrt{V_{3}}>4 n+3$. This requires a negative $V_{1}$, as was also seen in the cases $n=0,1,2$ above. If we now choose $V_{1}$ and $V_{3}$ from (47), the condition is translated to $s>1 / 4$, i.e., it becomes independant of $n$, and the exponent of $x^{2}$ is just $s-1 / 4$, i.e., with the $n$-dependent parametrization of $V_{1}$, we have $\lambda_{2 n}=\lambda_{0}=s-1 / 4$.

From (69), (73) and (77), we can also find the condition that has to be satisfied by $V_{-1}$ :

$$
V_{-1}=\frac{\left[V_{1}+(4 n+3) \sqrt{V_{3}}\right]\left[V_{1}+(4 n+5) \sqrt{V_{3}}\right]}{4 V_{3}} .
$$

With $V_{1}$ and $V_{3}$ from (47), we recover the corresponding $V_{-1}$ given by Ushveridze.

Finally, the relation between the energy and the coeffcients $c_{m}^{(n)}$ is $E_{2 n}=-4 \sqrt{V_{3}} c_{n-2}^{(n)} / c_{n}^{(n)}$, i.e., only the last two coefficients of the Taylor expansion of the wavefunction are required. This is clearly true when considering Eqs. (67), (71) and (75). This is also true to higher order $n$.

It is easy, although tedious, to go to higher $n$. For concision, the results are not written here.

This Section has described the sextic radial oscillator. If we suppress the centrifugial barrier, the system is defined on the whole real line: The potential corresponds to the sextic oscillator. This is the subject of the next Section.

\subsubsection{The sextic oscillator}

As in the previous Section, we restrict the analysis to $V_{2}=0$. We can solve the problem using the results of the sextic radial oscillator, implementing the constraint $V_{-1}=0$ by taking $s=1 / 4$ or $s=3 / 4$, i.e., $s=p / 2+1 / 4$, with $p$, the parity, being equal to 0 or 1 . The parametrization (47) leads to (64). Compared to the radial case, we now deal with odd or even eigenfunctions. This is due to the fact that the potential is real and symmetric around the origin. The case $s=1 / 4$ leads to a vanishing $\lambda_{N}$, hence deals with the even eigenfunctions. It corresponds to $p=0$. The case $s=3 / 4$, corresponding to $p=1$, implies $\lambda_{N}=1 / 2$. Starting with the sextic radial oscillator, defined on the positive half line, this leads to a prefactor $f(x)^{\lambda_{N}}=x$. Continuing the solution on the negative half line, we have finally generated the odd solutions.

Since the wavefunctions correspond either to $s=1 / 4$ or $s=3 / 4$, i.e., to two different potentials, the solutions fall into two different classes: For a given sextic oscillator, it is not possible to generate simultaneously both odd and even solutions.

\subsubsection{An exactly solvable case}

Even with $V_{-1}$ and $V_{-2}$ nonvanishing, the generalized sextic oscillator can be reduced to an exactly solvable model: Fixing the parameters in such a way to have $V(x)$ given by Eq. (65), 
all the solutions of the Schrödinger equation can be found.

Using our algorithm (17), we can see that, with $f(x)=1+x^{2}, h(x)=x$, we get $\lambda_{N}=$ $-1, g(x)=\exp \left(-x^{2} / 4\right)$. The general shape of the solution is

$$
\psi_{n}(x)=\frac{c_{n}}{1+x^{2}} \exp \left(-\frac{x^{2}}{4}\right) P_{n}\left(x^{2}\right)
$$

with $c_{n}$ a normalization coefficient and $P_{n}\left(x^{2}\right)$ a polynomial of $x^{2}$ of order $n$. The first few values are: $P_{0}=1, P_{1}=x\left(3+x^{2}\right), P_{2}=-1+2 x^{2}+x^{4}, P_{3}=x\left(-5+x^{4}\right)$. We see that a factor $x^{p}$ can be factored, with $p=0$ or $p=1$, leading again, as expected, to odd and even eigenfunctions. Our approach also shows that the energies are related one to each other by $E_{n+1}=E_{n}+1$, i.e., they are equally spaced like those of the harmonic oscillators.

In fact, using an irreducible second order Darboux transformation, we can relate the potential (65) to the potential of the harmonic oscillator. This implies that the eigenvalues of the potential of this Section are the same (up to a shift) as the ones of the harmonic oscillator, excepted that we lose its two first excited levels. The Darboux transformation also relates the corresponding eigenfunctions:

$$
\begin{aligned}
P_{0} & =1 \\
P_{n+1} & =x\left(3+x^{2}\right) H e_{n}(x)-\left(1+x^{2}\right) \frac{d H e_{n}(x)}{d x}
\end{aligned}
$$

with $H e_{n}(x)=2^{-n / 2} H_{n}(x / \sqrt{2})$ where $H_{n}(x)$ is the Hermite polynomial of order $n$.

It is trivial to check that the results coming from our approach satisfy this relation, and that our eigenvalues are the ones expected from the Darboux transformation. We note that since the Darboux transformation is constructed from the oscillator wavefunctions, the coefficients $V_{-1}, V_{-2}$ depend on the frequency $\omega$. In the case we have investigated here, the values $V_{-1}=$ $4, V_{-2}=-8$ correspond to the particular choice $V_{1}=1 / 4$, i.e., $\omega^{2}=1 / 4$.

\subsubsection{The Kuliy-Tkachuk potential}

We now turn to another class of problems: Those for which the number of solutions is fixed from the start. As an example, we choose the Kuliy-Tkachuk potential [7], for which three solutions can be found. It is a particular case of the potential (46), but with $V_{2}=V_{3}=0$. Before using the Kuliy-Tkachuk parameters given in Eq. (48), we first start with $V_{1}, V_{0}, V_{-1}, V_{-2}$ arbitrary. Compared to the general case of the sextic oscillator, our algorithm allows us to see that only one coefficient $V_{i}$, say $V_{-2}$, is constrained, the other ones being arbitrary. It also allows to see that the energies take simple values in terms of the parameters and $N$ :

$$
E_{N}=V_{0}+a V_{1}+\sqrt{b V_{1}}\left[(2 N+1)+4 \lambda_{N}\right], N=0,1,2, \ldots
$$

However, as $N$ is increased, the number of solution is growing because different values of the parameters are possible. For $N=0$ two solutions are possible: There are two possible values for $V_{-2}$ in terms of $a, b, V_{1}$. This leads to two possible values for $\lambda_{0}$, and then to two energies and two wavefunctions. Note that they correspond to two different values of $V_{-2}$, hence they do not refer to the same potential. The situation is similar for $N=1$. For bigger $N$, we face the same difficulty as in the case of the potential (46): Having taken $V_{2}=V_{3}=0$ has not 
simplified the solving of the problem, i.e., for general parameters, there is only one solution per given potential. On the other hand, fixing some of the parameters may lead to exactly solvable problems, such as in the previous Section, or to Q.E.S. systems with a fixed number of solutions : The K-T choice, with three solutions, corresponds to

$$
V_{1}=\frac{3}{4} b, V_{0}=\left(\frac{9}{4}-\frac{7}{2} \sqrt{3}\right) b, V_{-1}=2(3-\sqrt{3}) b, V_{-2}=(4 \sqrt{3}-6) b, a=1 .
$$

With this parametrization, we find, for $N=0$ :

$$
\begin{aligned}
\lambda_{0} & =\frac{\sqrt{3}}{1+\sqrt{3}}, \\
E_{0} & =0 \\
\psi_{0}(x) & =c_{0} \exp \left(-\frac{\sqrt{3}}{4} b x^{2}\right)\left(1+b x^{2}\right)^{\frac{\sqrt{3}}{1+\sqrt{3}}},
\end{aligned}
$$

while, for $N=1$, we have

$$
\begin{aligned}
\lambda_{1} & =\frac{\sqrt{3}}{3+\sqrt{3}}, \\
E_{1} & =3(2-\sqrt{3}) b \\
\psi_{1}(x) & =c_{1} \exp \left(-\frac{\sqrt{3}}{4} b x^{2}\right) x\left(1+b x^{2}\right)^{\frac{\sqrt{3}}{3+\sqrt{3}}} .
\end{aligned}
$$

The case $N=2$ leads to the same solution as the previous cases $N=0,1$, as well as to the new solution:

$$
\begin{aligned}
\lambda_{2} & =\frac{1}{2}(\sqrt{3}-1) \\
E_{2} & =2(3-\sqrt{3}) b \\
\psi_{2}(x) & =c_{2} \exp \left(-\frac{\sqrt{3}}{4} b x^{2}\right)\left(1-b x^{2}\right)\left(1+b x^{2}\right)^{\frac{1}{2}(\sqrt{3}-1)} .
\end{aligned}
$$

For higher $N$, we only generate the three cases above. As stated in the beginning of this Section, the Kuliy-Tkachuk potential corresponding to (85) allows to obtain exactly three wavefunctions and the corresponding eigenvalues which also satisfy the general relation (84).

Up to now, we have only considered the simplest variant (17) of our algorithm. We now discuss briefly two simple cases were the general approach (44)-(45) is needed. These cases are the Lamé equation and the screened Coulomb potential.

\subsection{The Lamé potential}

The Lamé equation is a Schrödinger equation with a periodic potential of the Elliptic Jacobic type:

$$
\left[-\frac{d^{2}}{d x^{2}}+k^{2} m_{N}\left(m_{N}+1\right) \operatorname{sn}^{2}(x, k)\right] \psi_{N}(x)=E_{N} \psi_{N}(x),
$$


with $k \in[0,1]$. This equation admits analytic solution for nonnegative integers $m_{N}$ [15]. Q.E.S. extensions of the Lamé equation can be found in the literature, e.g. [12]. In the following, we restrict ourselves to Eq. (95). This can be examined using our generalized algorithm (44)-(45). Several possibilities exist, showing the flexibility of our approach. For instance, we can choose:

$$
h(x)=\operatorname{sn}(x, k) \rightarrow h^{\prime}(x)=\operatorname{cn}(x, k) \operatorname{dn}(x, k)= \pm \sqrt{\left[1-h^{2}(x)\right]\left[1-k^{2} h^{2}(x)\right]} .
$$

The \pm sign implies to stay in a given interval, i.e. we choose the plus or minus sign in (96) the solution being extended appropriately on the whole line. Another choice corresponds to

$$
h(x)=\exp (i \phi) \rightarrow h^{\prime}(x)=i h(x) \sqrt{1+\frac{k^{2}}{4}\left[h(x)-\frac{1}{h(x)}\right]^{2}},
$$

with $\phi$ the amplitude of $x$. A third choice, which is the one we shall take to illustrate our algorithm, is

$$
\begin{aligned}
h(x) & =\frac{\operatorname{sn}(x, k)}{\operatorname{cn}(x, k)}, \\
\operatorname{sn}^{2}(x, k) & =\frac{h^{2}(x)}{1+h^{2}(x)}, \\
h^{\prime}(x) & =\sqrt{1+h^{2}(x)\left(2-k^{2}\right)+h^{4}(x)\left(1-k^{2}\right)},
\end{aligned}
$$

to which corresponds $f(x)=1 / \mathrm{sn}^{2}(x, k)=1+1 / h^{2}(x)$. Hence, we have $V \max =0, V \min =$ $2, V_{-2}=V_{0}=0, V_{-1}=k^{2} m_{N}\left(m_{N}+1\right), f_{l}^{0}=\delta_{l, 0}+\delta_{l,-2}, h_{l}=\delta_{l, 0}, \tilde{h}=\delta_{l, 0}+\left(2-k^{2}\right) \delta_{l, 2}+(1-$ $\left.k^{2}\right) \delta_{l, 4}$.

For $N=0$, our algorithm gives immediately $k=0$, or $k=1$ and/or $g(x)=1$. In fact, the two cases $k=0$ and $k=1$ are included as special case of the full $k$ problem, so that we can take immediately $g(x)=1$. For a compatibility with the remaining equations, our program shows that we need either $m=0, \lambda_{0}=0, E=0$, or $m=1, \lambda_{0}=-1 / 2, E=1+k^{2}$. The corresponding eigenfunctions are either a constant, or $\operatorname{sn}(x, k)$, respectively. In fact, because $\lambda_{0}=-1 / 2$, we see that the solution is $\psi(x)=[f(x)]^{-1 / 2}=|\operatorname{sn}(x, k)|$. However such a solution is not differentiable everywhere, and then is not a solution of the original Schrödinger equation. This implies that we need to consider the problem only on half a period of the function $\operatorname{sn}(x, k)$, thus allowing to remove the absolute operation, and to continue the solution on the whole real line, thus giving rise to $\psi(x)=\operatorname{sn}(x, k)$. This need of studying the problem on a given interval was also present in the choice (96). The need was seen from the beginning because of the \pm sign at the level of $h^{\prime}(x)$.

Putting the results together, we see that $N=0$ leads to

$$
\begin{aligned}
m_{0} & =0, \\
\lambda_{0} & =0, \\
E_{0} & =0, \\
\psi_{0}(x) & =c_{0},
\end{aligned}
$$

or

$$
m_{0}=1
$$




$$
\begin{aligned}
\lambda_{0} & =-\frac{1}{2}, \\
E_{0} & =1+k^{2}, \\
\psi_{0}(x) & =c_{0} \operatorname{sn}(x, k) .
\end{aligned}
$$

For $N=1$, several other new solutions are found:

$$
\begin{aligned}
m_{1} & =1, \\
\lambda_{1} & =-\frac{3}{2}, \\
E_{1} & =1, \\
\psi_{1}(x) & =c_{1} \operatorname{cn}(x, k),
\end{aligned}
$$

or

$$
\begin{aligned}
m_{1} & =2, \\
\lambda_{1} & =-1 \\
E_{1} & =4+k^{2}, \\
\psi_{1}(x) & =c_{1} \operatorname{cn}(x, k) \operatorname{sn}(x, k),
\end{aligned}
$$

or

$$
\begin{aligned}
m_{1} & =2 \\
\lambda_{1} & =-1 \\
E_{1} & =2\left(1+k^{2} \pm \sqrt{1-k^{2}+k^{4}}\right) \\
\psi_{0}(x) & =c_{1}\left[\left(1-k^{2}\right) \operatorname{sn}^{2}(x, k)+\left(k^{2} \mp \sqrt{1-k^{2}+k^{4}}\right) \mathrm{cn}^{2}(x, k)\right],
\end{aligned}
$$

or

$$
\begin{aligned}
m_{1} & =3 \\
\lambda_{1} & =-\frac{3}{2} \\
E_{1} & =\left(5+5 k^{2} \pm 2 \sqrt{4-7 k^{2}+4 k^{4}}\right) \\
\psi_{1}(x) & =c_{1} \operatorname{sn}(x, k)\left[\operatorname{sn}^{2}(x, k)\left(k^{2}-1\right)-\left(2 k^{2}-1 \mp \sqrt{4-7 k^{2}+4 k^{4}}\right)\right] .
\end{aligned}
$$

The solutions we got for $N=0$ were also obtained.

The analysis can of course be continued with higher values of $N$. What we observe is the expected set [15] of eight different types of solutions modulated by polynomials in sn.

\subsection{The screened Coulomb potential}

The screened Coulomb potential [16] is also a nice example of the working of our method. It was shown in that reference that, under a given relation between the coefficients of the potential $V(x)=F / x^{2}+G / x+H /\left(x+z^{2}\right)$, the solutions are polynomials. We shall verify this fact 
using our formalism. We first take $f(x)=\left(x+z^{2}\right)$ and $h(x)=x$ with $V_{k}=\delta_{k, 0}+\delta_{k,-1}$ and $v_{l, k}=0$ but for $v_{l, 0}=F \delta_{l,-2}+G \delta_{l,-1}$ and $v_{l,-1}=H \delta_{l, 0}$. We note that the coefficient $F$ has to be $F>-1 / 4$. This comes from the physical requirement of having, with the parametrization $F=L(L+1)+F_{1}$ and $L$ a nonvanishing integer (angular momentum), $F_{1}>-(L+1 / 2)^{2}$.

Starting our algorithm with $N=0$, we obtain straightforwardly that one of the coefficients of the potential must be expressed in terms of the others, e.g, G:

$$
G=\frac{4 F+(1+\sqrt{1+4 F})\left(4+H z^{2}\right)}{2 z^{2}} .
$$

Then, the solution can be expressed as

$$
\begin{aligned}
\lambda_{0} & =1 \\
E_{0} & =-\frac{4 F+2(1+\sqrt{1+4 F})\left(1+H z^{2}\right)+\left(H z^{2}\right)^{2}}{4 z^{4}}, \\
\psi_{0}(x) & =\exp \left[\frac{x}{2 z^{2}}\left(1+\sqrt{1+4 F}+H z^{2}\right)\right] x^{(1+\sqrt{1+4 F}) / 2}\left(x+z^{2}\right),
\end{aligned}
$$

with a positive argument of the square root from the condition $F>1 / 4$. Another solution is possible but we have rejected it because it is unbounded at the origin. We also observe the following relation between the energy, and the different coefficients of the potential:

$$
\frac{(G+H)^{2}}{-E_{0}}=(3+\sqrt{1+4 F})^{2} \text {. }
$$

In fact, the vanishing of the wavefunction at infinity requires $H z^{2}<1+\sqrt{1+4 F}$, see (128). Because of this, it is easy to verify that $(G+H)<0$. Hence, Eq. (129) can be replaced by

$$
\frac{(G+H)}{\sqrt{-E_{0}}}=-(3+\sqrt{1+4 F})
$$

a relation which was also obtained in [16], with the index $M=1$ in that reference.

The analysis can of course be done for higher $N$. The case $N=1$ is however already highly involved and will not be presented here. We just quote that, as in [16], the number 3 in Eq. (130) has to be replaced by $2 M+1$, with $M \geq 1$.

\section{A new Q.E.S. potential}

Using a Darboux transformation on the following sextic radial oscillator

$$
V(x)=\frac{1}{4} x^{6}-5 x^{2}+\frac{35}{4 x^{2}},
$$

we can show [14] that the wave function

$$
\psi(x)=\frac{\exp \left(-x^{4} / 8\right) x^{3 / 2}\left(6+x^{4}\right)}{x^{8}+4 x^{4}+20}
$$


is a solution of vanishing energy of the Schrödinger equation with the potential

$$
V(x)=\frac{1}{4} x^{6}+x^{2}+\frac{3}{4 x^{2}}+\frac{16 x^{2}\left(x^{4}-6\right)}{x^{8}+4 x^{4}+20}-\frac{2048 x^{6}}{\left(x^{8}+4 x^{4}+20\right)^{2}} .
$$

We show now this proposition using our algorithm, noting first that the potential obeys the parametrization $f(x)=x^{8}+4 x^{4}+20$ and $h(x)=x$. Moreover, $V_{k}=\delta_{k, 0}+\delta_{k,-1}+\delta_{k,-2}$ and $v_{l, k}=0$ but for $v_{l, 0}=3 \delta_{l,-2} / 4+\delta_{l, 2}+\delta_{l, 6} / 4, v_{l,-1}=-96 \delta_{l, 2}+16 \delta_{l, 6}$ and $v_{l,-2}=-2048 \delta_{l, 6}$.

The cases $N=0$ and $N=1$ do not lead to a solution. For $N=2$, all the constraints are fulfilled provided that $\tilde{g}_{l}^{1}=1 / 8 \delta_{l, 4}, \tilde{g}_{\log }^{1}=-7 / 2$, together with $c_{-1}^{(2)}=c_{1}^{(2)}=c_{0}^{(2)}=0, c_{-2}^{(2)}=$ $1 / 4\left(17-2 \tilde{g}_{\log }^{1}\right) c_{2}^{(2)}=6 c_{2}^{(2)}$, and $E_{2}=0, \lambda_{2}=1 / 8\left(\tilde{g}_{\log }^{1}-9 / 2\right)=-1$. The wavefunction is then

$$
\psi_{2}(x)=c_{2}^{(2)} \exp \left(-\frac{x^{4}}{8}\right) x^{7 / 2}\left(\frac{6}{x^{2}}+x^{2}\right)\left(x^{8}+4 x^{4}+20\right)^{-1},
$$

which is nothing else than (132).

\section{Conclusions}

We have proposed a general method for determining the analytic eigenfunctions (and associated eigenvalues) of a given (quasi) exactly solvable Schrödinger Hamiltonian. This method consists in a general algorithm replacing the differential Schrödinger equation by a finite set of algebraic equations, these ones being treated through a Mathematica implementation. Our general algorithm might be considered as relatively heavy from a purely technical point of view but it actually works straightforwardly. Moreover, for the majority of analytically solvable Schrödinger equations, it can be replaced by a simpler version as presented in Section 2. In all cases, it gives rise in a very systematic way to the solutions of the Schrödinger equations and also precises the conditions under which these equations are solvable. This systematization is particularly useful at the level of quasi exactly solvable Schrödinger equations for which one knew before our approach that some of them are relevant of $\operatorname{sl}(2, R)$ and the others not. These two different points of view have been unified through our formalism. We also assert that new quasi exactly solvable potentials (see Section 5) can be handled within our algorithm due to the fact that the group theoretical approach is not needed anymore.

We are also convinced that it is possible to extend our algorithm to the relativistic context, studying more particularly the quasi exactly solvable Dirac equations. Only a few studies, see for instance Ref. [17], deal with these equations because, by opposition to some of the Schrödinger ones, they are not in general subtended by a specific Lie (super) algebra. Our approach avoiding this group theoretical aspect should be of interest in the analysis of such Dirac equations. We plan to come back on these developments in the near future.

\section{Acknowledgments}

We thank Prof. Y. Brihaye for numerous useful discussions at various stages of this work and for interesting clarifications. We also thank Prof. B. Samsonov for several hints on Darboux transformations, and Prof. K. Penson for suggesting us the example of the screened Coulomb 
potential. The work of N. D. and B. VdB was supported by the Institut Interuniversitaire des Sciences Nucléaires de Belgique.

\section{References}

[1] M. Razavy, Am. J. Phys. 48 (1980) 285; Phys. Lett. A82 (1981) 7.

[2] A.V. Turbiner and A.G. Ushveridze, Phys.Lett. A126 (1987) 181.

[3] A.V. Turbiner, Comm. Math. Phys. 118 (1988) 467.

[4] A.G. Ushveridze, Quasi-Exactly Solvable Models in Quantum Mechanics, IOP Publishing Ltd (1994).

[5] V.M. Tkachuk, Phys.Lett. A245 (1998) 177.

[6] S.N. Dolya and O.B. Zaslavskii, J. Phys. A34 (2001) 1981.

[7] T.V. Kuliy and V.M. Tkachuk, J. Phys. A32 (1999) 2157.

[8] Y. Brihaye, N. Debergh and J. Ndimubandi, Mod. Phys. Let. A16 (2001) 1243.

[9] E. Witten, Nucl. Phys. B188 (1981) 513.

[10] Y. Brihaye and P. Kosinski, J. Math. Phys. 36 (1995) 4340.

[11] C. M. Bender and Q. Wang, A class of exactly-solvable eigenvalue problems, math$\mathrm{ph} / 0109007$.

[12] A. Khare, Phys. Lett. A288 (2001) 69.

[13] L. Skala, J. Cizek, J. Dvorak and V. Spirko, Phys. Rev. A53 (1996) 2009.

[14] N. Debergh, B.F. Samsonov and B. Van den Bossche, Darboux transformations for quasiexaclty solvable Hamiltonians, in preparation.

[15] F. M. Arscott, Periodic differential equations, Pergamon, Oxford (1964).

[16] M. Znojil, Phys. Lett. A94 (1983) 120.

[17] Y. Brihaye and P. Kosinski, Mod. Phys. Lett. A13 (1998) 1445. 